\title{
Influence of photoexcitation on the diamagnetic muonium states in Ge studied via their precession signatures
}

\author{
I. Fan, ${ }^{1, *}$ K. H. Chow ${ }^{1, \dagger}$ B. Hitti, ${ }^{2}$ R. Scheuermann, ${ }^{3}$ A. I. Mansour, ${ }^{1}$ W. A. MacFarlane, ${ }^{4}$ B. E. Schultz, ${ }^{1}$ M. Egilmez, ${ }^{1}$ \\ J. Jung, ${ }^{1}$ Y. G. Celebi, ${ }^{5}$ H. N. Bani-Salameh, ${ }^{6}$ B. R. Carroll, ${ }^{6}$ J. E. Vernon, ${ }^{6}$ and R. L. Lichti ${ }^{6}$ \\ ${ }^{1}$ Department of Physics, University of Alberta, Edmonton, Alberta, Canada T6G $2 G 7$ \\ ${ }^{2}$ TRIUMF, 4004 Wesbrook Mall, Vancouver, British Columbia, Canada V6T 2A3 \\ ${ }^{3}$ Paul Scherrer Institute, CH-5232 Villigen, Switzerland \\ ${ }^{4}$ Department of Chemistry, University of British Columbia, Vancouver, British Columbia, Canada V6T $1 Z 1$ \\ ${ }^{5}$ Department of Physics, Istanbul University, Beyazit 34459 Istanbul, Turkey \\ ${ }^{6}$ Department of Physics, Texas Tech University, Lubbock, Texas 79409-1051, USA
}

(Received 3 July 2008; published 28 October 2008)

\begin{abstract}
By using photoexcitation, we unambiguously establish that the well-known diamagnetic muonium spin precession signal in Ge is in fact due to two diamagnetic states. Their temperature dependences are studied up to room temperature. The signal due to one of these states is strongly influenced by the photoexcited carriers while the other is not. The identification of these two centers is discussed.
\end{abstract}

DOI: $10.1103 /$ PhysRevB.78.153203

Hydrogen is an important impurity that can change the structural, electrical, and optical properties of semiconductor devices because it passivates shallow dopants, platelets, etc., in the material. ${ }^{1}$ Since hydrogen is trapped very efficiently and rapidly, direct investigations on isolated hydrogen is very difficult. In fact, most of the experimental information on the isolated hydrogen comes from studies of highly spinpolarized muons via the muon spin rotation, relaxation, and resonance (i.e., $\mu \mathrm{SR}$ ) techniques (see Refs. 2-18, for example). The muon has a mass which is $\frac{1}{9}$ that of the proton, but both are much heavier than the electron. Since the reduced mass associated with the muonium $\left(\mu^{+} e^{-}\right)$atom is very similar to that of the hydrogen atom, they have very similar electronic structures. However, processes that are dependent on mass, such as diffusion, can be noticeably different.

It has been experimentally established that several distinct isolated muonium centers can be formed when $\mu^{+}$'s are implanted into germanium. Two of these states, commonly labeled as $\mathrm{Mu}_{\mathrm{BC}}^{0}$ and $\mathrm{Mu}_{T}^{0}$, are neutral and hence paramagnetic: the $\mathrm{Mu}_{\mathrm{BC}}^{0}$ center is located in the Ge-Ge bond center $(\mathrm{BC})$ position and, as a consequence of its location, has an anisotropic hyperfine interaction. The $\mathrm{Mu}_{T}^{0}$ center has an isotropic hyperfine interaction and is believed to be diffusing rapidly between interstitial tetrahedral $(T)$ sites. At low temperatures, approximately $\approx 72 \%$ of the implanted muons form $\mathrm{Mu}_{T}^{0}$ and $\approx 8 \%$ form $\mathrm{Mu}_{\mathrm{BC}}^{0}{ }^{2}$ The two neutral states both disappear at $\approx 110 \mathrm{~K}$ in high-purity germanium.

A nonparamagnetic ("diamagnetic") state is also observed in $\mathrm{Ge}$, corresponding to a muon without an unpaired electron. Possible candidates for this center include an isolated $\mathrm{Mu}^{-}$, isolated $\mathrm{Mu}^{+}$, and a muonium-dopant complex, but it is not obvious experimentally which of these states is the relevant one. This is because diamagnetic muonium centers lack a hyperfine interaction and, therefore, cannot be spectroscopically distinguished from each other. ${ }^{19}$ In the dark, the amplitude of the transverse-field $\mu$ SR diamagnetic precession signal is small at low temperatures but increases dramatically at $150-200 \mathrm{~K}^{2}$ Near room temperature, only the diamagnetic signal is present, and this signal accounts for almost $100 \%$ of the muon fraction.
PACS number(s): 71.20.Mq, 76.75.+i, 72.20.Jv

A particularly interesting study on Ge was carried out by Kadono et al. ${ }^{20,21}$ combining longitudinal-field (LF) $\mu \mathrm{SR}$ with the photoexcitation. The optical excitation generates free carriers in the semiconductor. By studying the so-called repolarization patterns, i.e., detailed field dependences of the LF- $\mu$ SR spectra, they concluded that there were two diamagnetic states coexisting in their Ge sample. One of these states underwent strong interactions with the photogenerated carriers while the other did not. They surmised that the former state was a muon undergoing charge exchange with carriers, while the latter was a complex between $\mathrm{Mu}^{-}$and a dopant, formed via a delayed reaction process. However, as described in Ref. 3, LF- $\mu$ SR studies have the disadvantage that it can be difficult to distinguish between $\mathrm{Mu}_{\mathrm{BC}}^{0}, \mathrm{Mu}_{T}^{0}$, and the diamagnetic muonium states which can be simultaneously present in the sample. Hence, it would be useful if one was able to carry out analogous studies under conditions where the diamagnetic state(s) can be unambiguously identified and investigated.

Recently, we demonstrated that optical illumination can affect the precession signals associated with the various muonium centers and used this technique to study the detailed photoinduced dynamics of muonium in $\mathrm{Si}^{22,23}$ The ability to monitor the precession signals is important since a unique set of precession frequencies is associated with each of the muonium states (as a consequence of their different hyperfine interactions), allowing unambiguous identification of the state involved in the dynamics. In this paper, we apply this technique to investigate the diamagnetic signal in Ge. While the precession signals associated with the diamagnetic states are indistinguishable in the dark, optical illumination shows that this signal is in fact composed of two different diamagnetic centers. The signal corresponding to one of these states is strongly affected by the free holes in the sample, while the other signal is hardly influenced by the free carriers. Possible candidates for these two diamagnetic states are discussed.

The optical $\mu \mathrm{SR}$ experiment was carried out at the M15 and M20 beamlines in TRIUMF, Vancouver, Canada. We will only briefly describe the experimental setup below-a 


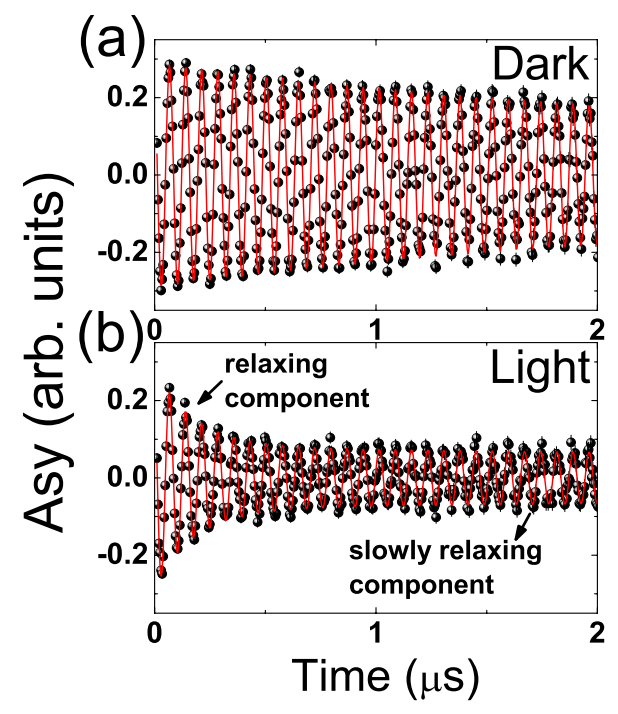

FIG. 1. (Color online) TF- $\mu$ SR spectra of diamagnetic muonium in Ge (sample n11) at $277 \mathrm{~K}$ (a) without illumination and (b) with 75-W light from the light bulb.

detailed description is available in Ref. 22. Four Ge samples (from Dr. Haller at Lawrence Berkeley Laboratory) with different dopant types and densities were investigated: n11 ( $n$ type $\left.\approx 10^{11} \mathrm{~cm}^{-3}\right), \mathrm{n} 13\left(n\right.$ type $\left.\approx 10^{13} \mathrm{~cm}^{-3}\right), \mathrm{p} 12(p$ type $\left.\approx 10^{12} \mathrm{~cm}^{-3}\right)$, and $\mathrm{p} 13\left(p\right.$ type $\left.\approx 10^{13} \mathrm{~cm}^{-3}\right)$. The sample under investigation was mounted in the optical cryostat, illuminated on one face, while the muons were implanted into the other face. The source of the illumination was a $250-\mathrm{W}$ tungsten halogen light bulb. The light was chopped with a mechanical chopper such that the sample was illuminated for $1 \mathrm{~s}$ and in the dark for $1 \mathrm{~s}$ (and the cycle repeated). This period keeps the temperature variation small and is still much longer than the recombination time of the photogenerated carriers. The light-on and light-off spectra were recorded in separate histograms. The diamagnetic states are detected as a standard TF- $\mu$ SR precession signal at the Larmor frequency of $\widetilde{\gamma}_{\mu} H$, where $\widetilde{\gamma}_{\mu}$ is the gyromagnetic ratio of the muon (135.54 MHz/T) and $H$ is the magnitude of the external magnetic field. The value of $H$ was chosen to be $\approx 1 \mathrm{kG}$, corresponding to a signal precessing at a convenient frequency of $\approx 13.5 \mathrm{MHz}$.

Figure 1 shows typical TF- $\mu$ SR spectra of the diamagnetic signal in Ge in the dark [Fig. 1(a)] and under optical illumination [Fig. 1(b)]. There is clearly a dramatic difference between the dark and illuminated spectra, a behavior that is in fact seen in all four Ge samples that we have studied. Note that in the dark, the spectrum appears to be a single diamagnetic signal that is relaxing [Fig. 1(a)]. However, when the sample is photoexcited, there is unambiguous evidence that this signal is in fact composed of two diamagnetic states. In particular, as shown in Fig. 1(b), one component or state undergoes significant relaxation while the other relaxes very little. We also investigated the influence of the light intensity (i.e., optical power was varied from 25 to $175 \mathrm{~W}$ ) on the relaxations and amplitudes of the two diamagnetic components. ${ }^{24}$ The amplitudes of both components did not depend on the intensity of the light. The relaxation rate of the

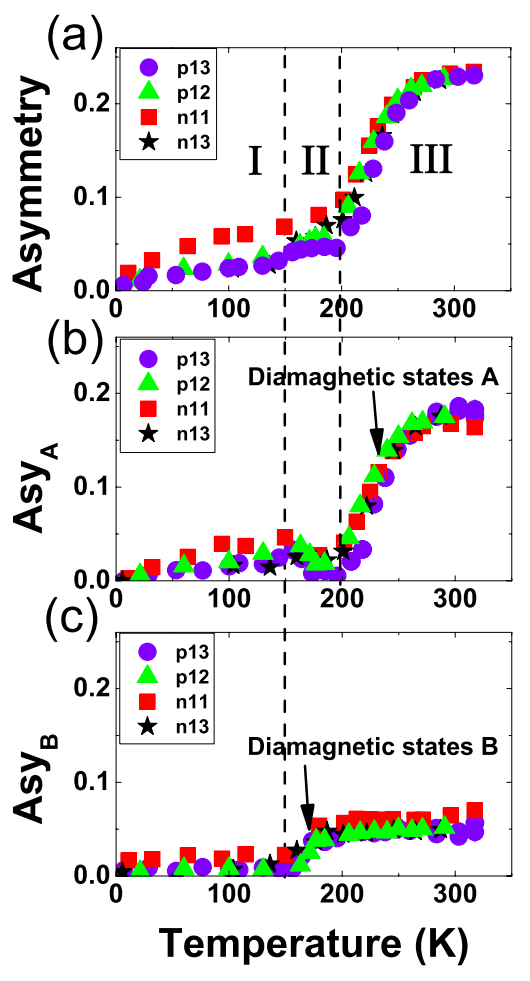

FIG. 2. (Color online) (a) The amplitude of the diamagnetic signal in the dark. The amplitudes of the diamagnetic states $A$ and $B$, separated using the photoexcitation technique, are shown in (b) and (c). See text.

relaxing diamagnetic signal increased significantly when the light intensity increased. However, the relaxation rate of the weakly relaxing diamagnetic signal only increased slightly.

There are several noteworthy conclusions related to the observations described thus far: (i) in order for a coherent precession to be observable in a TF- $\mu$ SR experiment, as in these experiments, both diamagnetic states must be formed promptly; i.e., they are formed at rates much faster than the precession frequency. The effect of the illumination is to generate free carriers in the Ge sample with which the muonium states may subsequently interact. Hence, the enhanced relaxation is due to a prompt diamagnetic state that is undergoing interactions with either the free electrons and/or holes, and the interaction rate increases as the carrier concentration increases. The nature of this interaction is discussed in more detail below. (ii) We can rule out a scenario whereby either diamagnetic state is "optically generated" since the brightness (i.e., optical power) of the light does not change the amplitude of either diamagnetic state. Therefore, the population of the diamagnetic states are still only determined by the thermalization process that follows the muon implantation.

The temperature dependence of the initial amplitude of the dark diamagnetic signal is shown in Fig. 2(a) for our four Ge samples. This behavior is similar to that observed in previous experiments. ${ }^{2}$ However, the current photoexcitation experiments enable us to clearly separate the contribution from each diamagnetic state and to monitor the temperature dependence of their amplitudes. This is done as follows: Designating $A$ and $B$ as the relaxing and slowly relaxing components, respectively, the TF- $\mu \mathrm{SR}$ polarization spectrum at 
each temperature (such as those shown in Fig. 1) is well fitted to the following function:

$$
\begin{aligned}
P(t)= & A s y_{A} \exp \left(-\lambda_{A} t\right) \cos (\omega t+\theta) \\
& +A s y_{B} \exp \left(-\lambda_{B} t\right) \cos (\omega t+\theta),
\end{aligned}
$$

where $A s y_{A}$ and $A s y_{B}$ denote the initial amplitudes (asymmetries) of the two diamagnetic states, $\lambda_{A}$ and $\lambda_{B}$ denote their relaxation rates, $\omega$ is the precession frequency, and $\theta$ is the phase. As discussed above, it is often difficult to distinguish the contributions of $A$ and $B$ to the dark signal. However, $A s y_{B}$ and $\lambda_{B}$ can be accurately obtained from the spectrum under the photoexcitation. (Recall that $A s y_{B}$ and $\lambda_{B}$ are not significantly affected by the illumination.) $A s y_{A}$ can then be obtained by subtracting $A s y_{B}$ from the total diamagnetic amplitude, i.e., the initial amplitude in the dark. These results are shown in Figs. 2(b) and 2(c).

Based on the temperature dependences of the amplitudes shown in Fig. 2, we divide the data into three regions. In region $\mathrm{I}(T<150 \mathrm{~K})$, the diamagnetic muonium fraction is small. In this region, most of the implanted muons form paramagnetic states, namely, $\mathrm{Mu}_{\mathrm{BC}}^{0}$ and $\mathrm{Mu}_{T}^{0}$, which subsequently disappear by $\approx 110 \mathrm{~K}^{2}{ }^{2}$ At the start of region II $(150 \mathrm{~K}<T<200 \mathrm{~K})$, the formation of diamagnetic state $B$ has become fast enough that significant amplitude can be observed [see Fig. 2(c)]. The amplitude of state $B$ saturates at $\approx 200 \mathrm{~K}$. Above $200 \mathrm{~K}$ (region III), diamagnetic state $A$ is being formed and the amplitude saturates by $\approx 280 \mathrm{~K}$. As mentioned earlier, this state undergoes significant interaction with the photogenerated charge carriers that leads to strong relaxation of the precession signal. Note that our observation that the amplitudes of both $A$ and $B$ are not significant until "high" temperatures indicate that these states arise from thermally activated transitions from neutral precursor states.

We now discuss in more detail the interactions of states $A$ and $B$, and also their possible identifications. As can be seen in Figs. 2(b) and 2(c), there is very little change in the amplitudes of $A$ and $B$ although the dopant type has changed from $n$ type to $p$ type, and the doping density has changed by several orders of magnitude. This suggests that neither state is a complex formed by muonium with the intentional electrically active impurity. By contrast, in addition to being significantly modified by the illumination, the dark relaxation rates of state $A$ are highly dependent on the doping of the sample. In particular, at a specific temperature, the relaxation rate of state $A$ increases as the free hole concentration increases, as shown in Fig. 3. On the other hand, increasing the free-electron concentration (cf. sample n11 with n13 in Fig. 3 ) does not increase the relaxation rate. This implies that state $A$ interacts with holes but not electrons and is, therefore, likely to be a negatively charged center. The relaxation associated with this state would then be due to a fluctuating hyperfine interaction that originates from charge-state fluctuations between the negatively charged and neutral states, e.g., $\mathrm{Mu}^{-}+h^{+} \rightleftharpoons \mathrm{Mu}^{0} .^{25}$ (A rapid charge cycle involving the $T$-site $\mathrm{Mu}^{0}$ has been observed in ultrapure $\mathrm{Ge},{ }^{26}$ also starting near $200 \mathrm{~K}$, although it was assigned to electron processes and $\mathrm{Mu}^{+}$.) Notice also that, as shown in Fig. 3, as the temperature increases in the $\mathrm{n} 11, \mathrm{n} 12$, and $\mathrm{p} 13$ samples, the re-

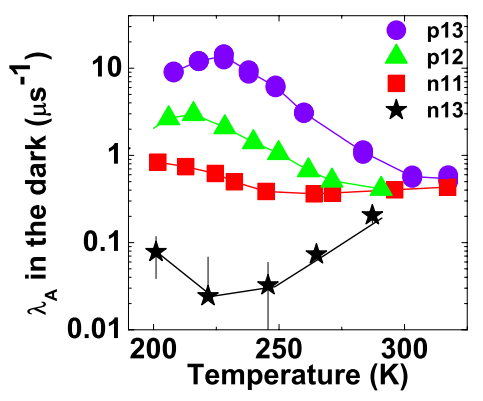

FIG. 3. (Color online) The dark relaxation of state $A$ in differently doped samples as a function of temperature. The lines are guides to the eyes.

laxation rate of state $A$ decreases. Such behavior indicates that state $A$ is in the so-called "dynamically narrowed" regime. ${ }^{23,25}$ That is, the transition rate from the paramagnetic precursor state is fast compared to its corresponding hyperfine oscillation(s) and becomes even faster as the temperature is increased. This is also consistent with the dramatic increase in state $A$ 's amplitude starting at $\approx 200 \mathrm{~K}$ (see Fig. 2), a signature that neutral muonium is making a rapid transition into the diamagnetic state ${ }^{2,26}$ in that temperature range.

The $\mathrm{Mu}^{-}$center is expected to be located in the $T$ site, at which the electron repulsion is the smallest among the diamond lattice. ${ }^{27}$ Hence, we assign state $A$ to be $\mathrm{Mu}_{T}^{-}$. Based on the large amplitude fraction of $\mathrm{Mu}_{T}^{-}$at high temperatures $[\approx 75 \%$ of the diamagnetic signal near room temperature corresponds to state $A$ (see Fig. 2)], it is reasonable to assign $\mathrm{Mu}_{T}^{0}$ as its precursor neutral state since it makes up the largest fraction of the low-temperature muonium fraction $(\approx 72 \%)$

We now discuss state $B$. We assign state $B$ to $\mathrm{Mu}_{\mathrm{BC}}^{+}$and its precursor state to isolated $\mathrm{Mu}_{\mathrm{BC}}^{0}$ (although this would mean that the low-temperature fraction of $\mathrm{Mu}_{\mathrm{BC}}^{0}$ in our samples is higher than the $8 \%$ reported in the literature ${ }^{2}$ ). In the dark, the relaxation of this state is small (generally $<0.05 \mu \mathrm{s}^{-1}$ in the dark for all four samples). The photoexcitation increases the relaxation rate of state $B$ slightly (up to about 0.1 to $0.7 \mu \mathrm{s}^{-1}$ depending on the sample and temperature), suggesting that there is some interaction with the photogenerated carriers, likely via the process $\mathrm{Mu}_{\mathrm{BC}}^{0} \rightleftharpoons \mathrm{Mu}_{\mathrm{BC}}^{+}+e^{-}$. This electron ionization or capture process is the analog of the well-established reaction involving $\mathrm{Mu}_{\mathrm{BC}}^{+}$in $\mathrm{Si}^{22,28-30}$ Note that a charge cycle at the $\mathrm{BC}$ site was also claimed to be relevant from previous LF relaxation data in ultrapure $\mathrm{Ge},{ }^{26}$ leading to a maximum relaxation rate of about $0.4 \mu \mathrm{s}^{-1}$ near $250 \mathrm{~K}$. This cycle became too rapid to have any visible effect just above $300 \mathrm{~K}$ as the intrinsic carrier concentration exceeds $\sim 10^{13} \mathrm{~cm}^{-3}$, roughly that estimated to be photogenerated in the present experiment.

We note that states $A$ or $B$ are not likely to be the $\mathrm{Mu}^{+}$-dopant complex. It is difficult to reconcile such an assignment with the very similar behavior of both states' amplitudes in four samples that have vastly different dopant concentrations and type. Furthermore, the complex should be quite stable to thermal dissociation, and therefore, charge fluctuations should not be relevant - this contradicts our observations that the relaxations of both states can be photoinduced. 
Finally, we compare our results on Ge with those of Kadono et al. $^{20}$ using the photoexcited LF- $\mu$ SR technique. As discussed earlier, they inferred the presence of two diamagnetic states. One of these states underwent strong interactions with the charge carriers-we believe that this is our state $A$. They assigned the other state to a $\mathrm{Mu}^{-}$complex that is formed in a delayed reaction process. This assignment of a delayed state is not consistent with our state $B$. In addition to our reasons outlined above making the assignment of state $B$ to a muonium-dopant complex unlikely, the fact that we can observe a precession signature is strong evidence that state $B$ is formed promptly. Their $n$-doped Ge sample had an estimated $[\mathrm{Sb}] \approx 10^{14} \mathrm{~cm}^{-3}$, which is a significantly higher $n$ doping than our highest $n$-type sample (n13). If their assign- ment is indeed correct, perhaps the formation of the complex becomes significantly more prominent as the $n$-dopant concentration increases.

In conclusion, using photoexcitation in combination with conventional TF- $\mu \mathrm{SR}$ allowed us to separate the diamagnetic signals in Ge. One of the diamagnetic components is likely $\mathrm{Mu}_{\mathrm{BC}}^{+}$and the other $\mathrm{Mu}_{T}^{-}$.

This research was partially supported by NSERC, the National Science Foundation (Grant No. DMR-0604501), and the R.A. Welch Foundation (Project No. D-1321). TRIUMF receives federal funding via a contribution through the National Research Council Canada. We thank R. Kadono and R. F. Kiefl for discussions. *ifan@phys.ualberta.ca

†kimchow@phys.ualberta.ca

${ }^{1}$ S. J. Pearton, J. W. Corbett, and T. S. Shi, Appl. Phys. A: Solids Surf. 43, 153 (1987); S. J. Pearton, J. W. Corbett, and M. Stavola, Hydrogen in Crystalline Semiconductors (Springer, New York,1992); S. M. Myers, M. I. Baskes, H. K. Birnbaum, J. W. Corbett, G. G. DeLeo, S. K. Estreicher, E. E. Haller, P. Jena, N. M. Johnson, R. Kirchheim, S. J. Pearton, and M. J. Stavola, Rev. Mod. Phys. 64, 559 (1992); S. K. Estreicher, Mater. Sci. Eng., R. 14, 319 (1995).

${ }^{2}$ B. D. Patterson, Rev. Mod. Phys. 60, 69 (1988).

${ }^{3}$ K. H. Chow, B. Hitti, and R. F. Kiefl, Semicond. Semimetals 51, 137 (1998).

${ }^{4}$ S. F. J. Cox, J. Phys.: Condens. Matter 15, R1727 (2003).

${ }^{5}$ K. H. Chow, R. F. Kiefl, W. A. MacFarlane, J. W. Schneider, D. W. Cooke, M. Leon, M. Paciotti, T. L. Estle, B. Hitti, R. L. Lichti, S. F. J. Cox, C. Schwab, E. A. Davis, A. Morrobel-Sosa, and L. Zavieh, Phys. Rev. B 51, 14762 (1995).

${ }^{6}$ K. H. Chow, B. Hitti, R. F. Kiefl, S. R. Dunsiger, R. L. Lichti, and T. L. Estle, Phys. Rev. Lett. 76, 3790 (1996).

${ }^{7}$ R. Kadono, A. Matsushita, K. Nagamine, K. Nishiyama, K. H. Chow, R. F. Kiefl, A. MacFarlane, D. Schumann, S. Fujii, and S. Tanigawa, Phys. Rev. B 50, 1999 (1994).

${ }^{8}$ K. H. Chow, B. Hitti, R. F. Kiefl, R. L. Lichti, and T. L. Estle, Phys. Rev. Lett. 87, 216403 (2001).

${ }^{9}$ B. E. Schultz, K. H. Chow, B. Hitti, Z. Salman, S. R. Kreitzman, R. F. Kiefl, and R. L. Lichti, Phys. Rev. B 72, 033201 (2005); B. E. Schultz, K. H. Chow, B. Hitti, R. F. Kiefl, R. L. Lichti, and S. F. J. Cox, Phys. Rev. Lett. 95, 086404 (2005); B. E. Schultz, I. Fan, B. Hitti, R. F. Kiefl, and K. H. Chow, Physica B (Amsterdam) 374-375, 464 (2006).

${ }^{10}$ K. Shimomura, R. Kadono, K. Ohishi, M. Mizuta, M. Saito, K. H. Chow, B. Hitti, and R. L. Lichti, Phys. Rev. Lett. 92, 135505 (2004).

${ }^{11}$ R. L. Lichti, W. A. Nussbaum, and K. H. Chow, Phys. Rev. B 70, 165204 (2004).

${ }^{12}$ K. H. Chow, R. F. Kiefl, B. Hitti, T. L. Estle, and R. L. Lichti, Phys. Rev. Lett. 84, 2251 (2000).

${ }^{13}$ R. C. Vilao, H. V. Alberto, J. P. Duarte, J. M. Gil, A. Weidinger, N. Ayres de Campos, R. L. Lichti, K. H. Chow, and S. F. J. Cox, Phys. Rev. B 72, 235203 (2005).

${ }^{14}$ K. H. Chow, R. L. Lichti, R. F. Kiefl, S. Dunsiger, T. L. Estle, B. Hitti, R. Kadono, W. A. MacFarlane, J. W. Schneider, D. Schu- mann, and M. Shelley, Phys. Rev. B 50, 8918 (1994).

${ }^{15}$ A. I. Mansour, Z. Salman, K. H. Chow, I. Fan, P. J. C. King, B. Hitti, J. Jung, and S. P. Cottrell, Phys. Rev. Lett. 100, 257602 (2008).

${ }^{16}$ R. C. Vilao, J. M. Gil, A. Weidinger, H. V. Alberto, J. Piroto Duarte, N. Ayres de Campos, R. L. Lichti, K. H. Chow, S. P. Cottrell, and S. F. J. Cox, Phys. Rev. B 77, 235212 (2008).

${ }^{17}$ R. L. Lichti, H. N. Bani-Salameh, B. R. Carroll, K. H. Chow, B. Hitti, and S. R. Kreitzman, Phys. Rev. B 76, 045221 (2007); R. L. Lichti, K. H. Chow, and S. F. J. Cox, Phys. Rev. Lett. 101, 136403 (2008).

${ }^{18}$ K. H. Chow, B. Hitti, and J. S. Lord, Phys. Rev. B 73, 113202 (2006).

${ }^{19}$ K. L. Hoffman, K. H. Chow, R. F. Kiefl, B. Hitti, T. L. Estle, and R. L. Lichti, Physica B (Amsterdam) 326, 175 (2003).

${ }^{20}$ R. Kadono, R. M. Macrae, K. Nishiyama, and K. Nagamine, Phys. Rev. B 55, 4035 (1997)

${ }^{21}$ R. Kadono, A. Matsushita, R. M. Macrae, K. Nishiyama, and K. Nagamine, Phys. Rev. Lett. 73, 2724 (1994).

${ }^{22}$ I. Fan, K. H. Chow, B. Hitti, R. Scheuermann, W. A. MacFarlane, A. I. Mansour, B. E. Schultz, M. Egilmez, J. Jung, and R. L. Lichti, Phys. Rev. B 77, 035203 (2008).

${ }^{23}$ I. Fan, K. H. Chow, R. Scheuermann, B. Hitti, W. A. MacFarlane, B. E. Schultz, A. I. Mansour, J. Jung, and R. L. Lichti, Physica B (Amsterdam) 401-402, 635 (2007).

${ }^{24}$ I. Fan, K. H. Chow, B. Hitti, A. I. Mansour, R. Scheuermann, W. A. MacFarlane, B. E. Schultz, M. Egilmez, J. Jung, Y. G. Celebi, H. N. Bani-Salameh, B. R. Carroll, J. E. Vernon, and R. L. Lichti Physica B (to be published).

${ }^{25}$ K. H. Chow, R. F. Kiefl, J. W. Schneider, B. Hitti, T. L. Estle, R. L. Lichti, C. Schwab, R. C. DuVarney, S. R. Kreitzman, W. A. MacFarlane, and M. Senba, Phys. Rev. B 47, 16004 (1993).

${ }^{26}$ R. L. Lichti, S. F. J. Cox, K. H. Chow, E. A. Davis, T. L. Estle, B. Hitti, E. Mytilineou, and C. Schwab, Phys. Rev. B 60, 1734 (1999).

${ }^{27}$ P. R. Briddon and R. Jones, Hyperfine Interact. 64, 593 (1991).

${ }^{28}$ S. R. Kreitzman, B. Hitti, R. L. Lichti, T. L. Estle, and K. H. Chow, Phys. Rev. B 51, 13117 (1995).

${ }^{29}$ B. Hitti, S. R. Kreitzman, T. L. Estle, E. S. Bates, M. R. Dawdy, T. L. Head, and R. L. Lichti, Phys. Rev. B 59, 4918 (1999).

${ }^{30}$ R. Kadono, R. M. Macrae, and K. Nagamine, Phys. Rev. B 68, 245204 (2003). 